\title{
The rotating molecular structures and the ionized outflow associated with IRAS 16547-4247
}

\section{Citation}

Franco-Hernández, Ramiro, James M. Moran, Luis F. Rodríguez, and Guido Garay. 2009. The rotating molecular structures and the ionized outflow associated with IRAS 16547-4247. The Astrophysical Journal 701, no. 2: 974-983. doi:10.1088/0004-637x/701/2/974.

\section{Published Version}

10.1088/0004-637x/701/2/974

\section{Permanent link}

http://nrs.harvard.edu/urn-3:HUL.InstRepos:34325453

\section{Terms of Use}

This article was downloaded from Harvard University's DASH repository, and is made available under the terms and conditions applicable to Other Posted Material, as set forth at http:// nrs.harvard.edu/urn-3:HUL.InstRepos:dash.current.terms-of-use\#LAA

\section{Share Your Story}

The Harvard community has made this article openly available.

Please share how this access benefits you. Submit a story.

Accessibility 


\title{
THE ROTATING MOLECULAR STRUCTURES AND THE IONIZED OUTFLOW ASSOCIATED WITH IRAS $16547-4247$
}

\author{
Ramiro Franco-Hernández ${ }^{1,2}$, James M. Moran ${ }^{1}$, Luis F. RodrígueZ ${ }^{2}$, and Guido Garay ${ }^{3}$ \\ ${ }^{1}$ Harvard-Smithsonian Center for Astrophysics, 60 Garden Street, Cambridge, MA 02138, USA; rfranco@ cfa.harvard.edu \\ ${ }^{2}$ Centro de Radioastronomía y Astrofísica, UNAM, Apartado Postal 3-72 (Xangari), 58089 Morelia, Michoacán, México \\ ${ }^{3}$ Departamento de Astronomía, Universidad de Chile, Camino el Observatorio 1515, Las Condes, Santiago, Chile \\ Received 2009 February 18; accepted 2009 June 18; published 2009 July 28
}

\begin{abstract}
We present Very Large Array $1.3 \mathrm{~cm}$ radio continuum and water maser observations as well as Submillimeter Array $\mathrm{SO}_{2}(226.300 \mathrm{GHz})$ and $1.3 \mathrm{~mm}$ dust continuum observations toward the massive star formation region IRAS 16547-4247. We find evidence of multiple sources in the central part of the region. There is evidence of a rotating structure associated with the most massive of these sources, traced at small scales $(\sim 50 \mathrm{AU})$ by the water masers. At large scales $(\sim 1000 \mathrm{AU})$, we find a velocity gradient in the $\mathrm{SO}_{2}$ molecular emission with a barely resolved structure that can be modeled as a rotating ring or two separate objects. The velocity gradients of the masers and of the molecular emission have the same sense and may trace the same structure at different size scales. The position angles of the structures associated with the velocity gradients are roughly perpendicular to the outflow axis observed in radio continuum and several molecular tracers. We estimate the mass of the most massive central source to be around 30 solar masses from the velocity gradient in the water maser emission. The main source of error in this estimate is the radius of the rotating structure. We also find water masers that are associated with the large-scale molecular outflow of the system, as well as water masers that are associated with other sources in the region. Our results suggest that the formation of this source, one of the most luminous protostars or protostellar clusters known, is taking place with the presence of ionized jets and disk-like structures.
\end{abstract}

Key words: stars: formation - stars: individual (IRAS 16547-4247)

\section{INTRODUCTION}

Our present understanding of star formation is primarily based on observations of the relatively abundant low-mass stars. The theoretical framework for star formation (Shu et al. 1987, 1993; see also McKee \& Ostriker 2007) has been successful in explaining the processes that occur in the formation of these lowmass stars, processes that are inferred from multiwavelength observations (e.g., Lada 1991; Evans 1999). Key ingredients in this scenario are the presence of a central protostar accreting from a circumstellar disk that is surrounded by an infalling envelope of dust and gas, as well as the presence of ionized jets and molecular outflows that remove angular momentum and mechanical energy from the accretion disk.

The applicability of this paradigm to the formation of massive stars remains unproven. It is possible that massive stars are formed by processes that are radically different from those that produce low-mass stars, such as by the merging of lower mass protostars (Bonnell et al. 1998). The role of the coalescence (Stahler et al. 2000) and accretion (Osorio et al. 1999; McKee \& Tan 2002) processes in the assembling of a massive star is still under debate. If massive $\mathrm{O}$ stars are formed by accretion we expect that disks and jets will be present during their earliest stages of evolution. On the other hand, if they are formed through coalescence of lower-mass stars then neither disks nor jets are expected since they would be disrupted during the merging process. For a recent review on the competing ideas to explain massive star formation see Zinnecker \& Yorke (2007).

Water masers have been observed in association with massive star formation regions and even though they have been studied for four decades (Cheung et al. 1969), their nature is still not fully understood. There has been a lot of discussion on where in the star formation region the physical conditions match those necessary for the excitation of the water masers. One idea is that the masers originate in a layer between the ionization and shock fronts in the expanding H II regions (e.g., Elitzur 1992). Another is that they are formed at the interface of the molecular material with the jets and outflows emanating from the forming stars (e.g., Furuya et al. 1999). Some observations also suggest that the water masers trace circumstellar disks (e.g., Torrelles et al. 2002).

IRAS $16547-4247$ is a luminous infrared source (bolometric luminosity of $6.2 \times 10^{4} L_{\odot}$ ), located at a distance of $2.9 \pm$ $0.6 \mathrm{kpc}$ (Rodríguez et al. 2008). It is thought to be associated with a massive young stellar object. Low angular resolution $\left(\sim 24^{\prime \prime}\right)$ millimeter wavelength observations show that the IRAS source is associated with an isolated and dense molecular core with a mass of $1.3 \times 10^{3} M_{\odot}$ and a radius of $0.2 \mathrm{pc}$ (Garay et al. 2003). Very Large Array (VLA) and Australia Telescope Compact Array (ATCA) interferometric observations of radio continuum at centimeter wavelengths show the presence of a thermal radio jet, located at the center of the core, and two lobes aligned and symmetrically separated from the jet by $\sim 10^{\prime \prime}$ or $\sim 0.14$ pc at a P.A. of $163^{\circ}$ (Garay et al. 2003; Rodríguez et al. 2005). In addition, observations of $\mathrm{H}_{2}$ emission at $2.12 \mu \mathrm{m}$ reveal a chain of knots of shock-excited gas extending over $1.5 \mathrm{pc}$, and $11.9 \mu \mathrm{m}$ continuum observations show a compact object $(\leqslant 0$." 8$)$ at the position of the jet (Brooks et al. 2003). The large-scale $\mathrm{H}_{2}$ flow and the radio jet are closely aligned, suggesting that these phenomena trace the outflowing gas at different distances from the forming star.

More recently Garay et al. (2007) observed the CO $J=$ $3 \rightarrow 2$ transition toward this source. Their observations show the presence of a collimated bipolar outflow roughly oriented in the north-south direction. The position angle of the molecular outflow (P.A. $=174^{\circ}$ ) is slightly different from the position angle of the radio jet $\left(\right.$ P.A. $\left.=167^{\circ}\right)$, suggesting that the jet axis is precessing. Additional evidence for precession has been 
presented by Rodríguez et al. (2008). Garay et al. (2007) estimated an inclination for the outflow of $i=84^{\circ} \pm 2^{\circ}$ from comparison of the position-velocity diagram of the outflowing CO with the biconical models of Cabrit et al. (1988). The momentum and energy parameters they derived are consistent with a massive young star driving the outflow. Also Garay et al. observed a strong signature of large-scale infall motions and derived an infall speed of $\sim 1 \mathrm{~km} \mathrm{~s}^{-1}$ and a mass infall rate of $\sim 1 \times 10^{-2} M_{\odot} \mathrm{yr}^{-1}$ at a radius of $\sim 0.2 \mathrm{pc}$.

Water masers were detected toward IRAS $16547-4247$ by Batchelor et al. (1980) with the Parkes radio telescope. They reported a flux of $100 \mathrm{Jy}$ and a $v_{\mathrm{LSR}}=-34 \mathrm{~km} \mathrm{~s}^{-1}$ for the maser emission. Later Forster \& Caswell (1989) made a brief VLA observation of IRAS $16547-4247$ for water masers. The angular resolution on their observations was enough to separate the masers into a few groups located near the central radio continuum source. However, due to their short integration time ( $\sim 8$ minutes) and narrow bandwidth, the radio continuum was not detected.

Here, we present water maser and radio continuum observations at $1.3 \mathrm{~cm}$ made with the VLA of the National Radio Astronomy Observatory (NRAO). ${ }^{4}$ We also present new observations made with the Submillimeter Array (SMA) ${ }^{5}$ of the $\mathrm{SO}_{2} 14(3,11)-14(2,12)$ transition at $226.300 \mathrm{GHz}$, as well as the $1.3 \mathrm{~mm}$ dust continuum emission. In Section 2 we describe the observations, while in Section 3 we present our results. In Section 4 we discuss the data and in Section 5 we summarize our conclusions.

\section{OBSERVATIONS}

The VLA observations were taken on 2007 June 7, using the correlator in the observing mode $2 \mathrm{AB}$. This allowed us to observe simultaneously the radio continuum and spectral line emission. The total bandwidth for the continuum was $25 \mathrm{MHz}$. For the spectral line observations we had a total of 64 channels with a resolution of $97.6 \mathrm{kHz}$ or $1.3 \mathrm{~km} \mathrm{~s}^{-1}$. These observations were taken in the A configuration, resulting in a beam with a FWHM of $0.31 \times 0$ '.07; P.A. $=0.8$ for ROBUST $=0$ weighting (Briggs 1995). The data were calibrated using the standard highfrequency calibration procedures for the VLA as described in the AIPS Cookbook. The phase calibrator was J1717-398 which had a bootstrapped flux of $0.30 \pm 0.01 \mathrm{Jy}$. A line channel with bright water maser emission was used to self-calibrate the line data and cross-calibrate the continuum data.

The SMA observations were taken on 2005 April 30. The array was in the extended configuration with a beam of FWHM of $2^{\prime \prime} .5 \times 0.97$; P.A. $=8^{\circ}$ (for a weighting with ROBUST $=0$ ), while the spectral resolution was $1.1 \mathrm{~km} \mathrm{~s}^{-1}$. The calibration was performed using MIR and MIRIAD. Callisto was used for flux calibration and 3C279 for bandpass calibration. The phase calibrator was 1745-290, with a bootstrapped flux of 3.15 Jy with a $20 \%$ accuracy. The continuum data at $1.3 \mathrm{~mm}$ were processed in MIRIAD using the task UVLIN in the lower sideband with $2 \mathrm{GHz}$ bandwidth centered at $217.1 \mathrm{GHz}$. The continuum was then self-calibrated in phase using CALIB in AIPS. The resulting calibration was then applied to the $\mathrm{SO}_{2}$ line data.

\footnotetext{
4 The NRAO is operated by Associated Universities, Inc. under cooperative agreement with the National Science Foundation.

5 The SMA is a joint project between the Smithsonian Astrophysical Observatory and the Academia Sinica Institute of Astronomy and Astrophysics and is funded by the Smithsonian Institution and the Academia Sinica.
}

\section{RESULTS}

\section{1. $1.3 \mathrm{~cm}$ VLA Observations}

Since IRAS $16547-4247$ is a rather southern source $\left(-42^{\circ}\right.$ in declination) the VLA had to observe through a large air mass during the whole observation run. This adversely affected the phase stability especially in poor weather. In the VLA observations presented by Rodríguez et al. (2005) the weather was not good and they used a self-calibration technique to obtain high dynamic range maps. Although the self-calibration largely improved the dynamic range, it did not improve the accuracy of the position of the source.

We made a special effort to determine the source position accurately. To obtain a better estimate for the position of the radio continuum we looked at the positions obtained from observations made in good weather where we could measure the position of the central bright radio continuum source. Rodríguez et al. (2005) reported positions derived from ATCA observations in 2003 February of $\alpha(2000)=16^{\mathrm{h}} 58^{\mathrm{m}} 17.216, \delta(2000)=-42^{\circ} 52^{\prime} 07^{\prime \prime} .64$ at $6 \mathrm{~cm}$ and $\alpha(2000)=16^{\mathrm{h}} 58^{\mathrm{m}} 17^{\mathrm{s}} .210, \delta(2000)=-42^{\circ} 52^{\prime} 07^{\prime \prime} .48$ at $3.6 \mathrm{~cm}$. The phase calibrator used in these observations was 1616-52. From the VLA archives there is data taken on 1993 January. The position measured from this epoch is $\alpha(2000)=16^{\mathrm{h}} 58^{\mathrm{m}} 17^{\mathrm{s}} .246, \delta(2000)=-42^{\circ} 52^{\prime} 07^{\prime \prime} .88$ at $3.6 \mathrm{~cm}$ with the phase calibrator $1626-298$. We have a position from the new VLA observations taken on 2006 May 31 and on June 8 (Rodríguez et al. 2008), that, after being concatenated, give the position $\alpha(2000)=16^{\mathrm{h}} 58^{\mathrm{m}} 17^{\mathrm{s}} .2093, \delta(2000)=$ $-42^{\circ} 52^{\prime} 07^{\prime \prime} .150$ at $3.6 \mathrm{~cm}$ with the phase calibrator 1626-298. Since the newest VLA data from 2006 have much more integration time than the snapshot from the archive we will just use the position from the former data, and from the two ATCA positions we take the position at $3.6 \mathrm{~cm}$ because the $6 \mathrm{~cm}$ data have lower angular resolution. We adopt as the final position estimate for the central bright radio continuum source at centimeter wavelengths the average of the ATCA position at $3.6 \mathrm{~cm}$ (Rodríguez et al. 2005 ) and the most recent (2006) VLA position (Rodríguez et al. 2008). This gives $\alpha(2000)=16^{\mathrm{h}} 58^{\mathrm{m}} 17^{\mathrm{s}} .210 \pm 0.001, \delta(2000)=$ $-42^{\circ} 52^{\prime} 07^{\prime \prime} .32 \pm 00^{\prime} .17$, where the errors quoted are one half the difference between the two observations used.

We used self-calibration techniques to obtain a final image for the new observations at $1.3 \mathrm{~cm}$ presented here. We assume that the position of the peak $1.3 \mathrm{~cm}$ emission coincides with our adopted position. Since the water masers were observed simultaneously with the continuum, their positions are also referenced to the same coordinates. It is worthwhile to note that the relative positions between the water masers and the $1.3 \mathrm{~cm}$ continuum are very accurate and independent of the absolute position adopted in the self-calibration. The image of the $1.3 \mathrm{~cm}$ continuum emission is shown in Figure 1. The emission is dominated by the thermal jet, for which we derive, from a Gaussian ellipsoid fit made using the task JMFIT of AIPS, a total flux density of $10.9 \pm 1.3 \mathrm{mJy}$ and deconvolved angular dimensions of $0^{\prime \prime} .55 \pm 0^{\prime \prime} .08 \times 0^{\prime \prime} .13 \pm 0^{\prime \prime} .03$ with P.A. of $177^{\circ} \pm 4^{\circ}$. The position angle of the major axis is consistent with that derived at longer wavelengths (Garay et al. 2003; Rodríguez et al. $2005,2008)$, indicating that at $7 \mathrm{~mm}$ we are tracing the ionized jet. The image shows two marginal $(4 \sigma)$ components to the south of the jet that require confirmation in deeper images.

The distribution of the main features of the IRAS 16547-4247 region, including that of the different water maser groups is shown in Figure 2. The parameters of the 


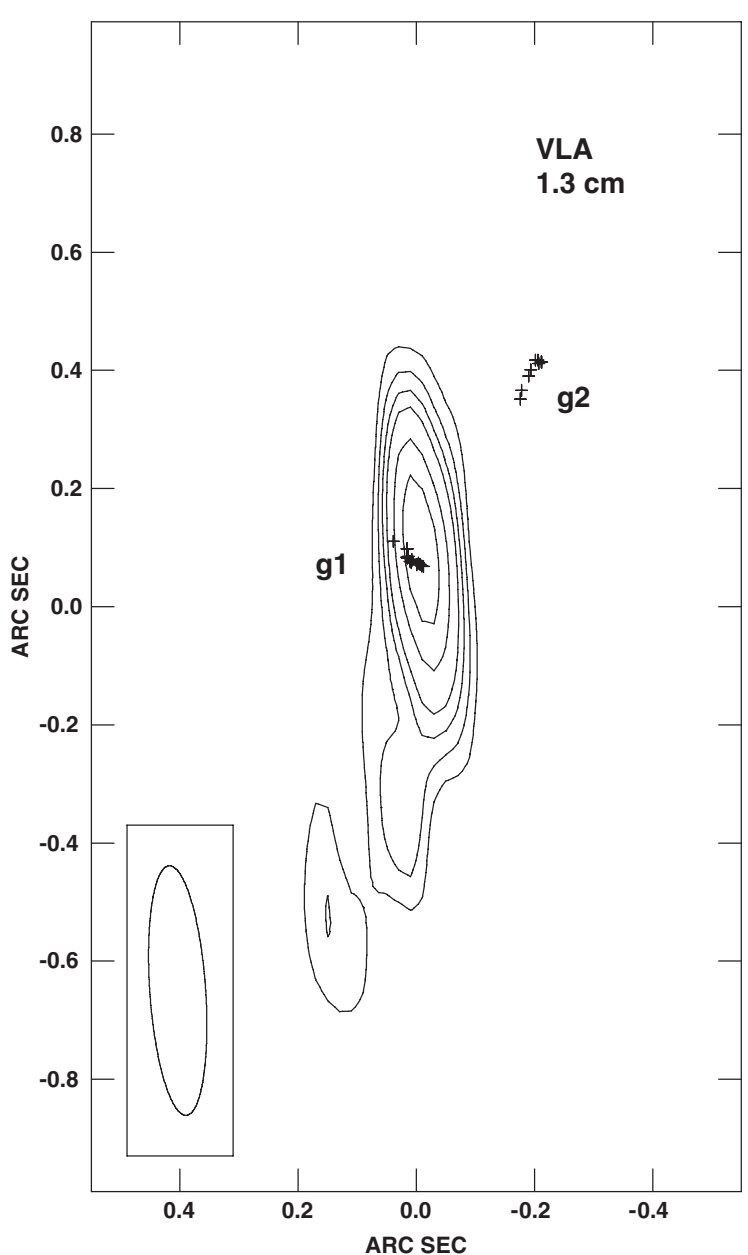

Figure 1. VLA $1.3 \mathrm{~cm}$ continuum emission from the IRAS $16547-4247$ central source. The image was made with ROBUST $=5$ weighting. Contours are $-4,-3,3,4,5,6,8$, and 10 times $0.42 \mathrm{mJy}^{\text {beam }}{ }^{-1}$, the rms noise of the map. The half-power contour of the beam $\left(0^{\prime \prime} 43 \times 0^{\prime \prime} 09 ; 4^{\circ}\right)$, is shown in the bottom left corner. The peak position adopted for this source is $\alpha(2000)=16^{\mathrm{h}} 58^{\mathrm{m}} 17^{\mathrm{s}} \cdot 210, \delta(2000)=-42^{\circ} 52^{\prime} 07^{\prime \prime} 32$. The main component is marginally resolved and elongated along P.A. of $177^{\circ}$. The small crosses mark the positions of the water masers in the compact structures located in groups $\mathbf{g 1}$ and $\mathbf{g 2}$.

individual maser spots are listed in Table 1. It is clear that there are several groups of masers associated with different radio continuum sources seen in the $3.6 \mathrm{~cm}$ continuum map from Rodríguez et al. (2005). These groups are separated with the horizontal lines in Table. 1. The strongest masers, in the group marked as g1, are associated with the central and brightest radio continuum source. Their radial velocities range from $-25.3 \mathrm{~km} \mathrm{~s}^{-1}$ to $-54.2 \mathrm{~km} \mathrm{~s}^{-1}$. Most of the masers in the $\mathbf{g 1}$ group form a compact structure extending in the east-west direction (see Figure 3). There is another clear group of masers denoted $\mathbf{g} 2$ in Figure 2. This group is located to the north-west of the radio continuum peak and the maser radial velocities extend from $-30.5 \mathrm{~km} \mathrm{~s}^{-1}$ to $-42.4 \mathrm{~km} \mathrm{~s}^{-1}$. Most of the masers in the $\mathbf{g} 2$ group form a compact structure that is shown in Figure 4. We will discuss these two compact structures below.

\subsection{SMA $1.3 \mathrm{~mm}$ Continuum Observations}

The SMA dust continuum map presented in Figure 5 clearly shows that the emission at $1.3 \mathrm{~mm}$ exhibits a morphology that looks like two partially resolved sources separated by about 2.'0. We fitted the emission to two Gaussian ellipsoids with the AIPS task JMFIT. The parameters resulting from the fit (positions,

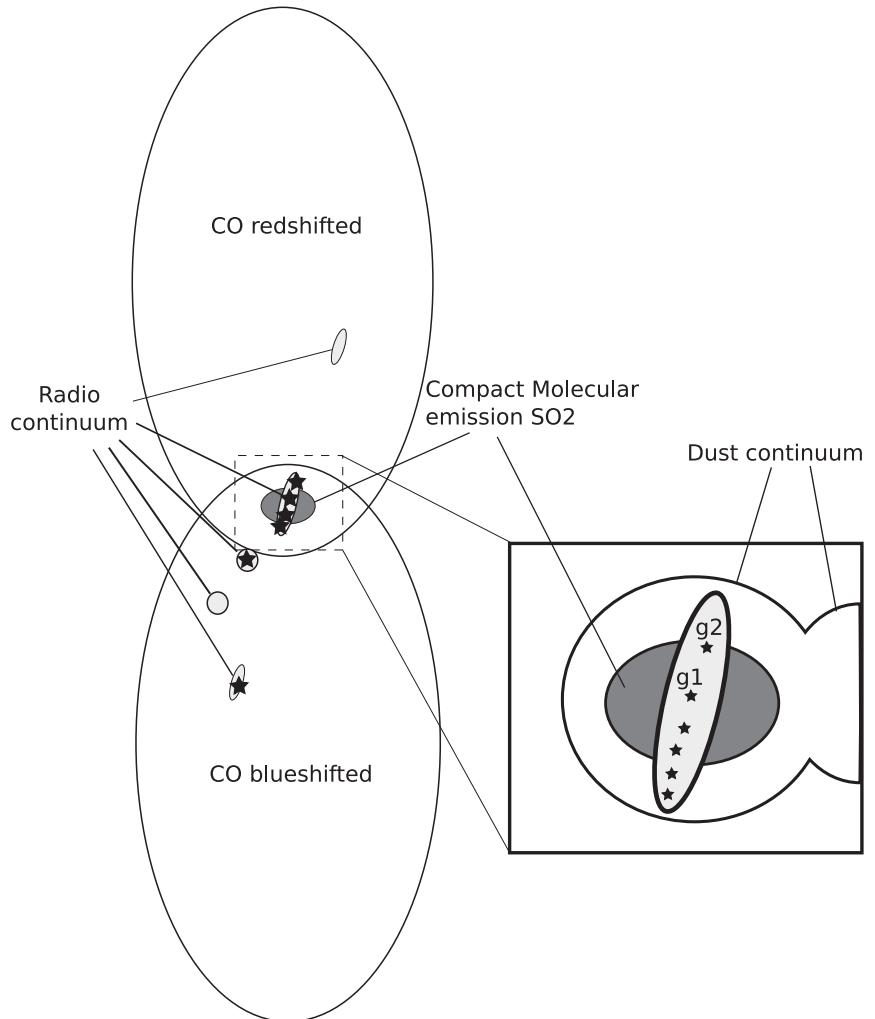

Figure 2. Schematic (not to scale) showing the different features and components of IRAS 16547-4247. The stars mark the positions of water maser groups.

flux densities, deconvolved angular sizes, and position angles) are given in Table 2. From the fit we find that, as expected from Figure 5, the eastern component is the brightest in the continuum. This component is also detected in several molecular lines (R. Franco-Hernández et al. 2010, in preparation). The total dust continuum flux is $1.03 \mathrm{Jy}$, split in 0.81 and $0.22 \mathrm{Jy}$ for the eastern and western components, respectively. Following Chini et al. (1987), assuming a constant temperature of $300 \mathrm{~K}$ (appropriate for dust at $1000 \mathrm{AU}$ from a $6 \times 10^{4} L_{\odot}$ source), optically thin emission with a dust to gas ratio of 0.01 , and a dust mass opacity of $1 \mathrm{~cm}^{2} \mathrm{~g}^{-1}$ at $1.3 \mathrm{~mm}$, we calculate a total gas mass in the double dust continuum source of $\sim 6 M_{\odot}$, split into 4.7 and $1.3 M_{\odot}$ for the strong and weak components, respectively.

The western component is unresolved, while the deconvolved angular sizes (FWHM) obtained from the fit for the eastern component are $1^{\prime \prime} .34 \times 0.84$; P.A. $=107^{\circ}$. The stronger $1.3 \mathrm{~mm}$ component can be interpreted as a flattened structure, approximately in the east-west direction with a size of $\sim 3500 \mathrm{AU}$. This structure may be tracing a large disk around the central source or sources.

It is interesting to note that the west (secondary) $1.3 \mathrm{~mm}$ component appears to be a source different from the centimeter source D (Rodríguez et al. 2008), which also appears to the west of the main source. While the west $1.3 \mathrm{~mm}$ source is displaced by $1^{\prime \prime} .94 \pm 0.15$ from the main source, the centimeter source D is displaced by $1^{\prime \prime} .02 \pm 0$.'03 from the main source. We conclude that there are at least three sources within $2^{\prime \prime}$ of the main source: the main source itself, the centimeter source $\mathrm{D}$, and the west $1.3 \mathrm{~mm}$ source reported here.

Finally, we note that the centimeter position adopted for the main source, $\alpha(2000)=16^{\mathrm{h}} 58^{\mathrm{m}} 17^{\mathrm{s}} .210 \pm 0.001 ; \delta(2000)=$ $-42^{\circ} 52^{\prime} 07^{\prime \prime} .32 \pm 0^{\prime} .17$, and discussed in Section 3.1, does not 
Table 1

Parameters of Individual Water Maser Spots

\begin{tabular}{|c|c|c|c|c|c|c|c|}
\hline $\begin{array}{c}V_{\mathrm{LSR}} \\
\left(\mathrm{km} \mathrm{s}^{-1}\right)\end{array}$ & $\begin{array}{c}\alpha(\mathrm{J} 2000) \\
16^{\mathrm{h}} 58^{\mathrm{m}}\end{array}$ & $\begin{array}{l}\Delta \alpha \\
(\mathrm{s})\end{array}$ & $\begin{array}{l}\delta(\mathrm{J} 2000) \\
-42^{\circ} 52^{\prime}\end{array}$ & $\begin{array}{c}\Delta \delta \\
(\operatorname{arcsec})\end{array}$ & $\begin{array}{l}\text { Flux } \\
(\mathrm{Jy})\end{array}$ & $\begin{array}{c}\Delta \text { Flux } \\
(\mathrm{Jy})\end{array}$ & $\begin{array}{c}\text { Continuum } \\
\text { Association }^{\mathrm{a}}\end{array}$ \\
\hline-12.1 & 17.46426 & 0.00043 & 16.4344 & 0.0018 & 0.37 & 0.01 & S-1 \\
\hline-13.4 & 17.46383 & 0.00029 & 16.4380 & 0.0013 & 0.50 & 0.01 & \\
\hline-14.7 & 17.46567 & 0.00011 & 16.4222 & 0.0005 & 1.79 & 0.01 & \\
\hline-16.0 & 17.46614 & 0.00006 & 16.4183 & 0.0002 & 8.69 & 0.03 & \\
\hline-17.4 & 17.46617 & 0.00005 & 16.4183 & 0.0002 & 11.94 & 0.04 & \\
\hline-18.7 & 17.46618 & 0.00005 & 16.4185 & 0.0003 & 5.17 & 0.02 & \\
\hline-20.0 & 17.46631 & 0.00014 & 16.4204 & 0.0006 & 1.05 & 0.01 & \\
\hline-21.3 & 17.46628 & 0.00009 & 16.4199 & 0.0004 & 1.87 & 0.01 & \\
\hline-22.6 & 17.46628 & 0.00009 & 16.4200 & 0.0004 & 1.62 & 0.01 & \\
\hline-23.9 & 17.46628 & 0.00023 & 16.4202 & 0.0011 & 0.60 & 0.01 & \\
\hline-27.9 & 17.36690 & 0.00469 & 10.1078 & 0.0066 & 0.04 & 0.03 & B \\
\hline-30.5 & 17.39604 & 0.00215 & 10.1549 & 0.0102 & 0.50 & 0.07 & \\
\hline-31.8 & 17.39619 & 0.00083 & 10.1587 & 0.0037 & 1.50 & 0.08 & \\
\hline-33.2 & 17.39632 & 0.00050 & 10.1617 & 0.0023 & 1.72 & 0.06 & \\
\hline-34.5 & 17.39644 & 0.00090 & 10.1576 & 0.0041 & 0.62 & 0.03 & \\
\hline-37.1 & 17.41415 & 0.00095 & 09.8128 & 0.0183 & 0.43 & 0.04 & \\
\hline-38.4 & 17.37794 & 0.00123 & 10.0880 & 0.0052 & 0.20 & 0.02 & \\
\hline-39.7 & 17.37802 & 0.00008 & 10.0873 & 0.0004 & 2.73 & 0.01 & \\
\hline-41.1 & 17.37802 & 0.00004 & 10.0865 & 0.0002 & 5.99 & 0.02 & \\
\hline-42.4 & 17.37801 & 0.00004 & 10.0862 & 0.0002 & 4.27 & 0.01 & \\
\hline-43.7 & 17.37801 & 0.00022 & 10.0857 & 0.0010 & 0.71 & 0.01 & \\
\hline-18.7 & 17.22278 & 0.02314 & 08.4282 & 0.0450 & 0.08 & 0.03 & Jet \\
\hline-20.0 & 17.21856 & 0.00050 & 08.4653 & 0.0021 & 0.31 & 0.01 & \\
\hline-21.3 & 17.21879 & 0.00030 & 08.4696 & 0.0013 & 0.64 & 0.01 & \\
\hline-27.9 & 17.22072 & 0.00064 & 08.4688 & 0.0027 & 1.85 & 0.07 & \\
\hline-29.2 & 17.22069 & 0.00015 & 08.4801 & 0.0006 & 9.79 & 0.09 & \\
\hline-30.5 & 17.22080 & 0.00010 & 08.4815 & 0.0005 & 12.96 & 0.08 & \\
\hline-37.1 & 17.21056 & 0.00969 & 08.2030 & 0.0106 & 1.49 & 0.13 & \\
\hline-29.2 & 17.22741 & 0.00068 & 07.9606 & 0.0030 & 2.66 & 0.10 & Jet \\
\hline-30.5 & 17.22792 & 0.00003 & 07.9472 & 0.0001 & 36.83 & 0.07 & \\
\hline-31.8 & 17.22794 & 0.00002 & 07.9463 & 0.0001 & 68.62 & 0.09 & \\
\hline-33.2 & 17.22800 & 0.00003 & 07.9450 & 0.0001 & 38.77 & 0.06 & \\
\hline-34.5 & 17.22906 & 0.00032 & 07.9280 & 0.0008 & 5.94 & 0.05 & \\
\hline-35.8 & 17.21846 & 0.01244 & 07.5844 & 0.0204 & 8.60 & 0.31 & \\
\hline-37.1 & 17.22580 & 0.00115 & 07.6553 & 0.0046 & 1.58 & 0.06 & \\
\hline-50.3 & 17.22169 & 0.00005 & 07.8103 & 0.0002 & 2.93 & 0.01 & \\
\hline-51.6 & 17.22173 & 0.00002 & 07.8109 & 0.0001 & 10.09 & 0.02 & \\
\hline-52.9 & 17.22173 & 0.00002 & 07.8115 & 0.0001 & 9.51 & 0.02 & \\
\hline-54.2 & 17.22169 & 0.00006 & 07.8124 & 0.0003 & 2.27 & 0.01 & \\
\hline-29.2 & 17.20965 & 0.00002 & 07.3098 & 0.0000 & 85.18 & 0.09 & Jet \\
\hline-31.8 & 17.21571 & 0.00013 & 07.8589 & 0.0005 & 15.11 & 0.10 & \\
\hline-33.2 & 17.21588 & 0.00004 & 07.8572 & 0.0002 & 25.63 & 0.06 & \\
\hline-34.5 & 17.21453 & 0.00004 & 07.7631 & 0.0002 & 31.66 & 0.05 & \\
\hline-35.8 & 17.21287 & 0.00002 & 07.6567 & 0.0001 & 39.31 & 0.05 & \\
\hline-37.1 & 17.21284 & 0.00002 & 07.6550 & 0.0001 & 30.19 & 0.04 & \\
\hline-38.4 & 17.21275 & 0.00006 & 07.6365 & 0.0003 & 6.50 & 0.02 & \\
\hline-50.3 & 17.22121 & 0.00005 & 07.8130 & 0.0002 & 3.39 & 0.01 & Jet \\
\hline-51.6 & 17.22114 & 0.00003 & 07.8134 & 0.0001 & 11.59 & 0.02 & \\
\hline-52.9 & 17.22113 & 0.00003 & 07.8135 & 0.0001 & 10.93 & 0.02 & \\
\hline-54.2 & 17.22112 & 0.00006 & 07.8135 & 0.0003 & 2.58 & 0.01 & \\
\hline-25.3 & 17.21011 & 0.00009 & 07.3292 & 0.0004 & 2.14 & 0.01 & Jet (g1) \\
\hline-26.6 & 17.20985 & 0.00002 & 07.3261 & 0.0001 & 17.31 & 0.03 & \\
\hline-27.9 & 17.20958 & 0.00002 & 07.3286 & 0.0001 & 52.47 & 0.07 & \\
\hline-29.2 & 17.20936 & 0.00002 & 07.3309 & 0.0001 & 69.73 & 0.09 & \\
\hline-30.5 & 17.20909 & 0.00004 & 07.3316 & 0.0002 & 38.43 & 0.08 & \\
\hline$-31.8^{b}$ & 17.20338 & 0.00095 & 07.3008 & 0.0017 & 13.47 & 0.19 & \\
\hline$-33.2^{\mathrm{b}}$ & 17.20076 & 0.00020 & 07.3157 & 0.0008 & 6.89 & 0.07 & \\
\hline$-34.5^{\mathrm{b}}$ & 17.21109 & 0.00066 & 07.4804 & 0.0013 & 38.92 & 0.18 & \\
\hline$-38.4^{\mathrm{b}}$ & 17.21141 & 0.00005 & 07.3695 & 0.0003 & 7.09 & 0.02 & \\
\hline-39.7 & 17.21092 & 0.00006 & 07.3200 & 0.0003 & 3.73 & 0.01 & \\
\hline-41.1 & 17.21093 & 0.00011 & 07.3250 & 0.0005 & 2.38 & 0.02 & \\
\hline-42.4 & 17.21091 & 0.00009 & 07.3224 & 0.0004 & 2.27 & 0.01 & \\
\hline
\end{tabular}


Table 1

(Continued)

\begin{tabular}{lccccccc}
\hline \hline $\begin{array}{l}V_{\text {LSR }} \\
\left(\mathrm{km} \mathrm{s}^{-1}\right)\end{array}$ & $\begin{array}{c}\alpha(\mathrm{J} 2000) \\
16^{\mathrm{h}} 58^{\mathrm{m}}\end{array}$ & $\begin{array}{c}\Delta \alpha \\
(\mathrm{s})\end{array}$ & $\begin{array}{c}\delta(\mathrm{J} 2000) \\
-42^{\circ} 52^{\prime}\end{array}$ & $\begin{array}{c}\Delta \delta \\
(\operatorname{arcsec})\end{array}$ & $\begin{array}{c}\text { Flux } \\
(\mathrm{Jy})\end{array}$ & $\begin{array}{c}\Delta \text { Flux } \\
(\mathrm{Jy})\end{array}$ & $\begin{array}{c}\text { Continuum } \\
\text { Association }^{\mathrm{a}}\end{array}$ \\
\hline-43.7 & 17.21095 & 0.00007 & 07.3254 & 0.0003 & 2.39 & 0.01 & \\
-45.0 & 17.21099 & 0.00010 & 07.3241 & 0.0004 & 1.48 & 0.01 & \\
-46.3 & 17.21117 & 0.00019 & 07.3236 & 0.0008 & 0.813 & 0.01 & \\
-47.6 & 17.21156 & 0.00016 & 07.3175 & 0.0006 & 1.08 & 0.01 & \\
-48.9 & 17.21165 & 0.00011 & 07.3164 & 0.0005 & 1.56 & 0.01 & \\
-50.3 & 17.21143 & 0.00015 & 07.3147 & 0.0006 & 1.49 & 0.01 & \\
-51.6 & 17.21162 & 0.00058 & 07.3019 & 0.0021 & 1.07 & 0.03 & \\
-52.9 & 17.21375 & 0.00280 & 07.2891 & 0.0081 & 0.989 & 0.05 & \\
-54.2 & 17.21159 & 0.00104 & 07.3020 & 0.0041 & 0.294 & 0.01 & \\
\hline$-30.5^{\mathrm{b}}$ & 17.20013 & 0.00090 & 07.2632 & 0.0030 & 8.05 & 0.14 & Jet $(\mathbf{g} 2)$ \\
-31.8 & 17.19417 & 0.00070 & 07.0484 & 0.0025 & 6.58 & 0.14 & \\
-33.2 & 17.19286 & 0.00041 & 07.0098 & 0.0016 & 4.86 & 0.08 & \\
-34.5 & 17.19396 & 0.00074 & 07.0338 & 0.0027 & 2.58 & 0.06 & \\
-35.8 & 17.19256 & 0.00073 & 06.9989 & 0.0030 & 1.69 & 0.06 & \\
-37.1 & 17.19132 & 0.00029 & 06.9885 & 0.0012 & 2.60 & 0.04 & \\
-38.4 & 17.19088 & 0.00007 & 06.9862 & 0.0003 & 3.60 & 0.02 & \\
-39.7 & 17.19097 & 0.00005 & 06.9853 & 0.0002 & 4.63 & 0.01 & \\
-41.1 & 17.19144 & 0.00006 & 06.9828 & 0.0002 & 4.49 & 0.02 & \\
-42.4 & 17.19186 & 0.00008 & 06.9827 & 0.0004 & 2.42 & 0.01 & \\
\hline
\end{tabular}

Notes.

a Radio continuum source (from Rodríguez et al. 2008) associated with the group of masers.

b Spot not forming part of the compact structures discussed in the text.

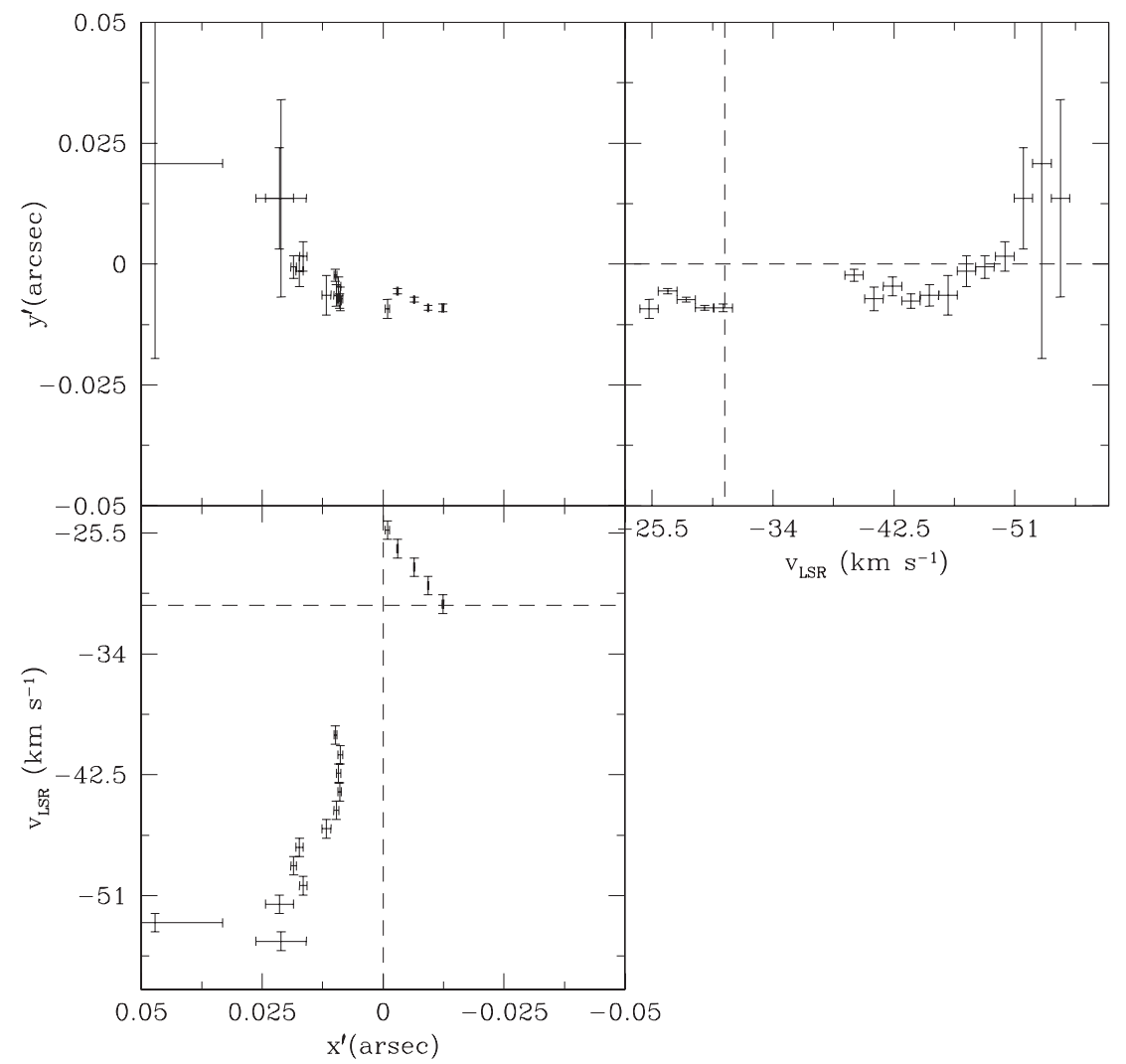

Figure 3. Distribution in position and radial velocity of the compact structure of masers in $\mathbf{g} 1$ (Figure 2). The upper left panel shows the positions of the masers with respect to the peak of the radio continuum at $1.3 \mathrm{~cm}$. The upper right and lower panels are the position-velocity diagrams for the same masers. The broken lines show the position of the radio continuum and the LSR velocity of the source in their respective axes. The positions have been rotated $13^{\circ}$ counterclockwise to make the ordinate parallel to the ionized jet. The errors in position shown are 10 times larger than the real values.

coincide within the errors with the position obtained from the $1.3 \mathrm{~mm}$ image (see Table 2), $\alpha(2000)=16^{\mathrm{h}} 58^{\mathrm{m}} 17.247 \pm$ $0.002 ; \delta(2000)=-42^{\circ} 52^{\prime} 08^{\prime \prime} \cdot 09 \pm 0.04$ and that these positions differ by $\sim 0$ '" 87 . We tentatively attribute this discrepancy to the use of different phase calibrators. To facilitate modeling of the region we assume that the SMA $1.3 \mathrm{~mm}$ peak coincides with 


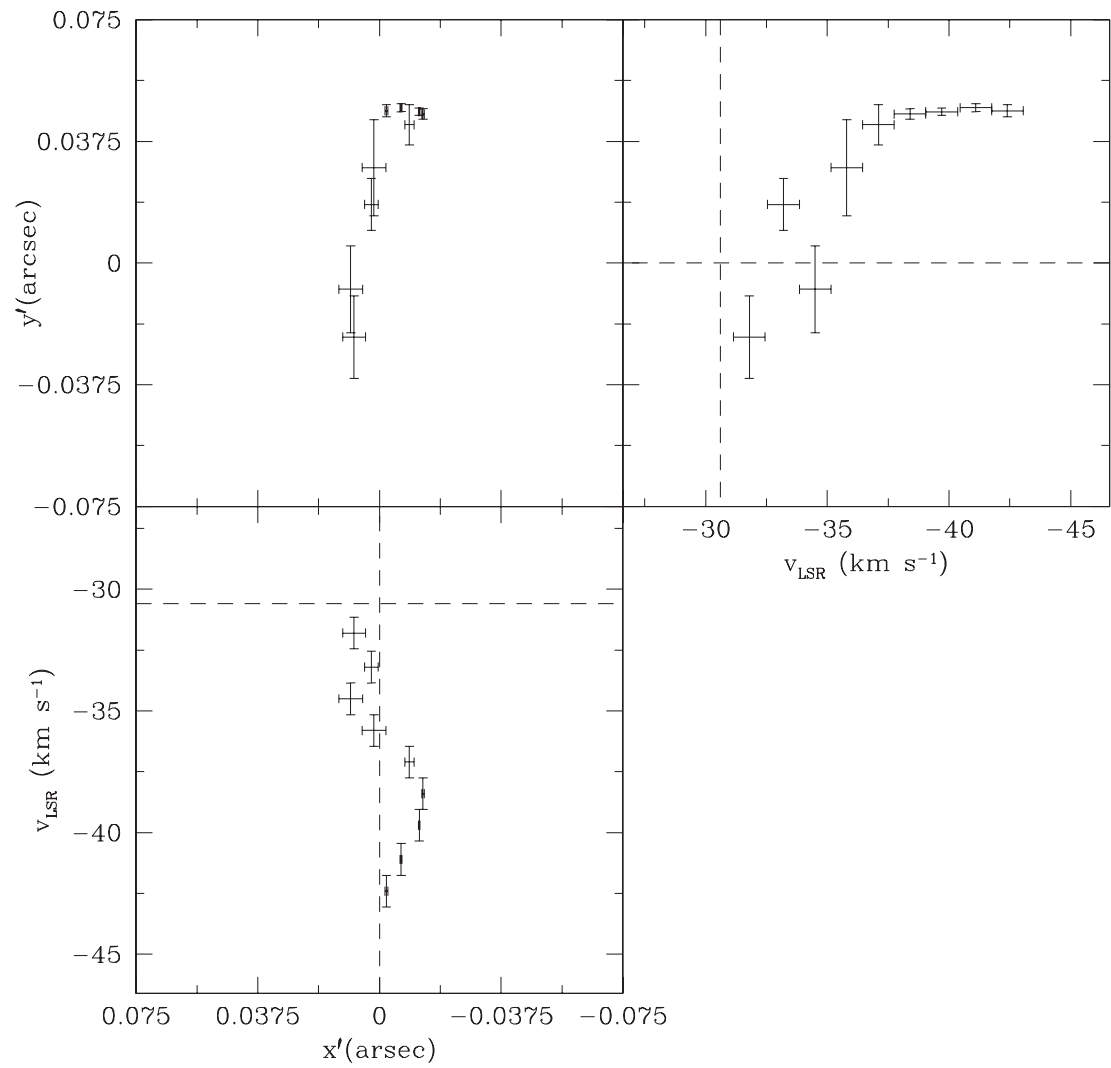

Figure 4. Distribution in position and radial velocity of the compact structure of masers in $\mathbf{g} 2$ (Figure 2). Panels are as in Figure 3. A clear gradient can be seen to the north of the peak of radio continuum. Reference position is $\alpha(J 2000)=16^{\mathrm{h}} 58^{\mathrm{m}} 07^{\mathrm{s}} \cdot 1930, \delta(J 2000)=-42^{\circ} 52^{\prime} 7^{\prime \prime} .028$. The positions have been rotated $13^{\circ}$ counterclockwise to make the ordinate parallel to the ionized jet. The errors in position shown are 10 times larger than the real values.

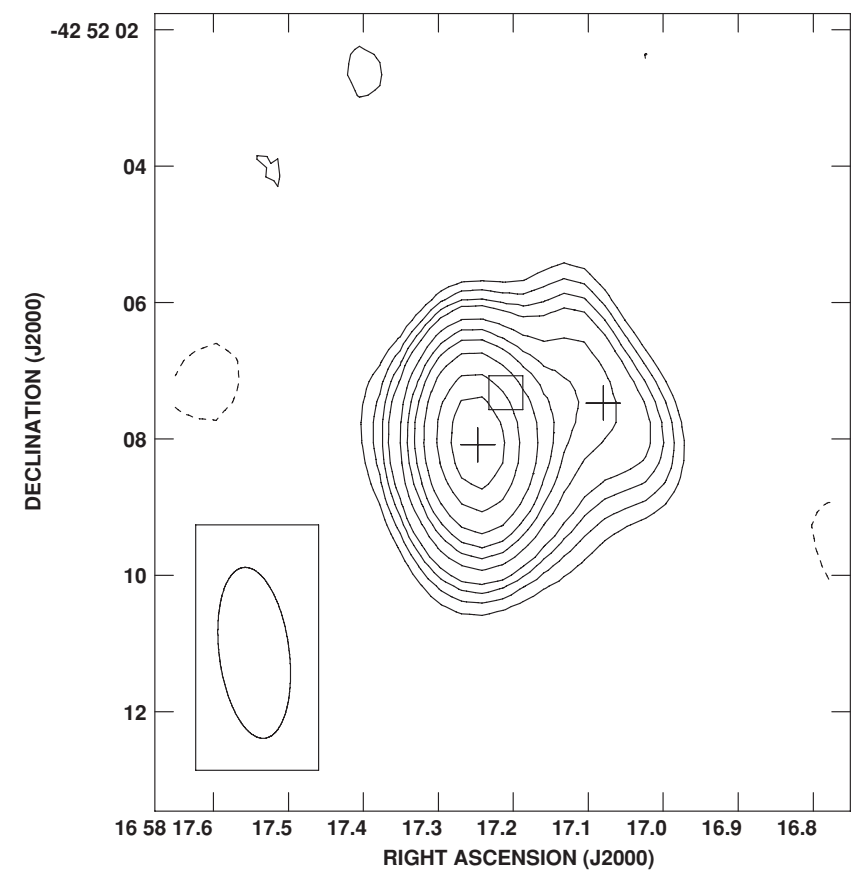

Figure 5. SMA $1.3 \mathrm{~mm}$ dust continuum emission from the IRAS $16547-4247$ central source. Contours are $-4,-3,3,4,5,6,8,10,12,15,20$, and 25 times $16 \mathrm{mJy}^{\text {beam }}{ }^{-1}$, the rms noise of the map. The crosses mark the position of the two components obtained from the fit to the image, whose parameters are given in Table 2 . The square box marks the position finally adopted for the peak $1.3 \mathrm{~mm}$ emission (see discussion in the text).

the VLA/ATCA $3.6 \mathrm{~cm}$ peak. The same offset is applied to the SMA molecular data.

\section{3. $\mathrm{SMA} \mathrm{SO}_{2}$ Observations}

We detected strong $\mathrm{SO}_{2}$ emission toward the dominant eastern dust component, but not toward the weaker western component. Figure 6 shows the first moment of the $\mathrm{SO}_{2} 226.300 \mathrm{GHz}$ transition together with the $1.3 \mathrm{~mm}$ continuum emission, both overlaid on the VLA $3.6 \mathrm{~cm}$ emission. The $\mathrm{SO}_{2}$ emission comes from a structure of $\sim 1500 \mathrm{AU}$ in radius. We measured the peak position in each velocity channel of the $\mathrm{SO}_{2}$ emission. In Table 3, we list these positions and they are plotted in Figure 7. In this plot, it can be seen that the velocity gradient is roughly perpendicular to the direction of the outflow observed in CO (Garay et al. 2007) and the radio jet (Garay et al. 2003; Rodríguez et al. 2005; this paper).

\section{DISCUSSION \\ 4.1. Water Masers}

In Figure 3, we show the relative positions of the water masers in the compact structure in group $\mathbf{g} 1$ with respect to the radio continuum at $1.3 \mathrm{~cm}$. It can be seen that the position of the peak of the radio continuum lies close to the position of the structure. From the lower left panel of Figure 3, it is tempting to infer that the LSR velocity for the source is around $-42 \mathrm{~km} \mathrm{~s}^{-1}$ since this is the central velocity for this group of masers and would make them look like a complete rotating ring with masers red and blueshifted with respect to this velocity. However, this velocity is $12 \mathrm{~km} \mathrm{~s}^{-1}$ blueshifted with respect to the LSR velocity measured from the molecular lines. This difference is large compared with the radial dispersion velocity in young stellar clusters which is 

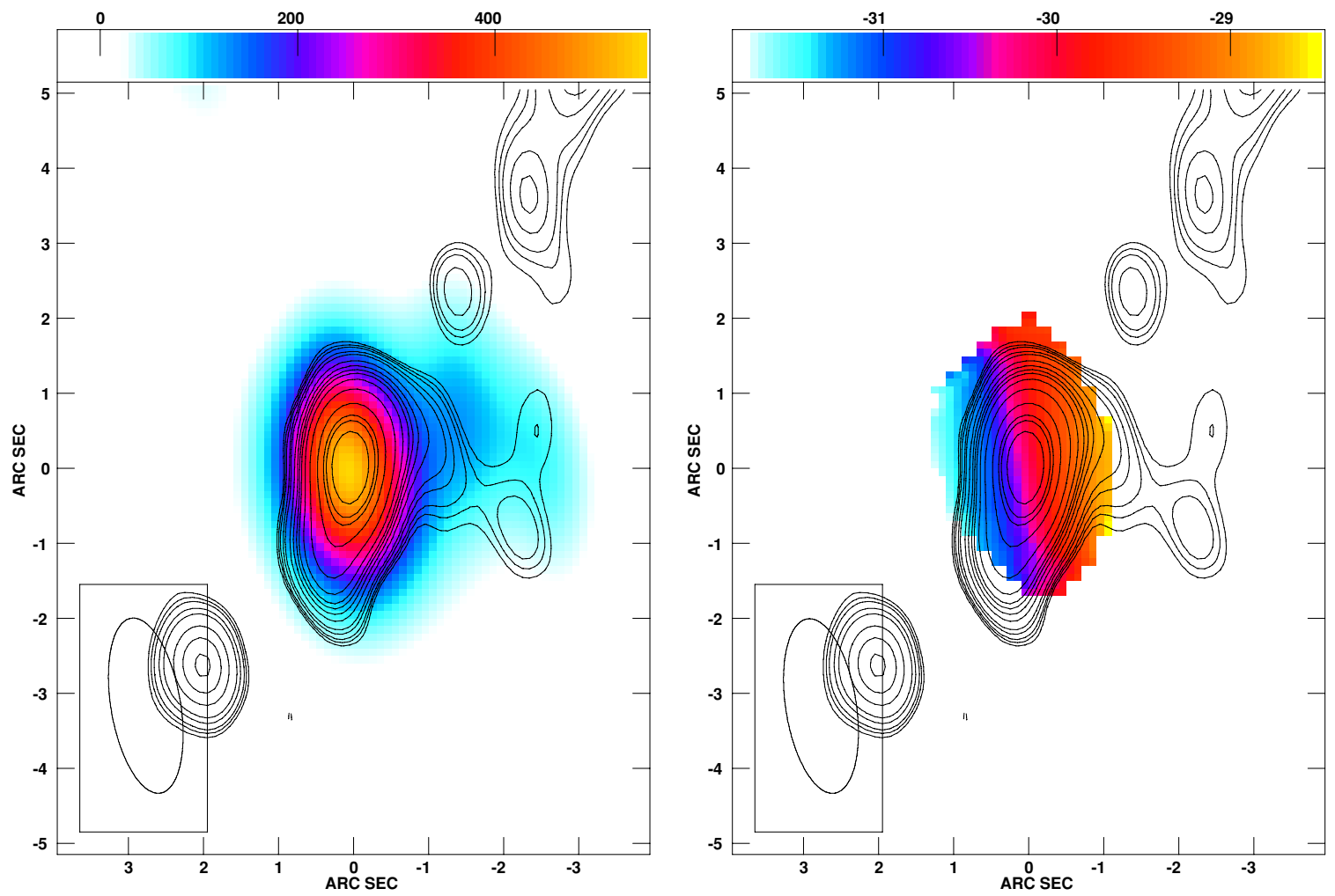

Figure 6. Left panel shows the $1.3 \mathrm{~mm}$ dust continuum emission in color and the $3.6 \mathrm{~cm}$ free-free radio continuum in contours. The color bar at the top shows the color coding for the $230 \mathrm{GHz}$ flux density in mJy beam ${ }^{-1}$. Contour levels for the $3.6 \mathrm{~cm}$ emission are $-5,5,8,10,15,20,40,60,80,100,140,180$ times the rms noise level of $30 \mu \mathrm{Jy}$ beam $^{-1}$. The $3.6 \mathrm{~cm}$ source at the lower left corner of the image most probably traces an independent star and is not associated

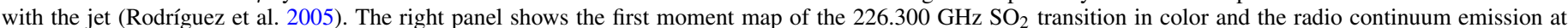
$3.6 \mathrm{~cm}$ with the same contours as in the left panel (Rodríguez et al. 2005). The color bar at the top shows the color coding for the LSR velocity of the gas in km s ${ }^{-1}$. Note that the western component is not detected in the $\mathrm{SO}_{2}$ emission (right panel). The synthesized beam for the $3.6 \mathrm{~cm}$ data is shown in the bottom left corner of each panel. The synthesized beam for the $230 \mathrm{GHz}$ data is $2.3 \times 0.8$; P.A. $=11^{\circ}$.

Table 2

Continuum Components at $1.3 \mathrm{~mm}$

\begin{tabular}{|c|c|c|c|c|}
\hline \multirow[t]{2}{*}{ Component } & \multicolumn{2}{|c|}{ Position $^{\mathrm{a}}$} & \multirow{2}{*}{$\begin{array}{c}\text { Total Flux } \\
\text { Density (mJy) }\end{array}$} & \multirow[t]{2}{*}{ "Deconvolved Angular Size ${ }^{\mathrm{b}}$} \\
\hline & $\alpha(\mathrm{J} 2000)$ & $\delta(\mathbf{J} 2000)$ & & \\
\hline West & $17.080 \pm 0.006$ & $07.47 \pm 0.12$ & $220 \pm 35$ & $\leqslant 2^{\prime \prime} .2 \times \leqslant 1^{\prime \prime} .5 ;+45^{\circ} \pm 29^{\circ}$ \\
\hline East & $17.247 \pm 0.002$ & $08.09 \pm 0.04$ & $810 \pm 37$ & $1^{\prime \prime} .34 \pm 0^{\prime \prime} .10 \times 0^{\prime \prime} .84 \pm 0^{\prime \prime} .33 ;+107^{\circ} \pm 17^{\circ}$ \\
\hline
\end{tabular}

Notes.

${ }^{\mathrm{a}}$ Units of right ascension are seconds with respect to $16^{\mathrm{h}} 58^{\mathrm{m}}$ and units of declination are arcseconds with respect to $-42^{\circ} 52^{\prime}$.

$\mathrm{b}$ The values given are major axis $\times$ minor axis; position angle of major axis. For the east component, we only obtained upper limits to the angular size.

expected to be only a few $\mathrm{km} \mathrm{s}^{-1}$ (e.g., $\sim 2 \mathrm{~km} \mathrm{~s}^{-1}$ for the center of the Orion Nebula, Jones \& Walker, 1988; as well as for Cyg OB2, Kiminki et al. 2007).

If the velocity center of the $\mathbf{g} \mathbf{1}$ compact structure is at the LSR velocity of $-30.6 \mathrm{~km} \mathrm{~s}^{-1}$ we can still interpret it as a rotating torus around the central source. However, in this case we have emission coming from masers mainly blueshifted with respect to the LSR velocity. The lack or weak emission from one side of the disk (blueshifted or redshifted) has been observed in other sources (Val'Tts et al. 2005) and it is probably related to different excitation conditions in different parts of the ring. Assuming that the central source is at the position marked by the peak of the radio continuum and that the maser emission is coming from a ring in a Keplerian orbit, we can estimate the central mass.

We first obtain the gradient in velocity adjusting a straight line to the masers in the velocity range from -38.4 to $-54.2 \mathrm{~km} \mathrm{~s}^{-1}$.
For this fit, we discard the maser at $-52.9 \mathrm{~km} \mathrm{~s}^{-1}$ which is very noisy and seems to depart from the rest of the features. We obtain a slope of $d V / d \theta=-812 \pm 71 \mathrm{~km} \mathrm{~s}^{-1} \operatorname{arcsec}^{-1}$ and an intercept of $-35 \pm 1 \mathrm{~km} \mathrm{~s}^{-1}$. In the previous expression $V$ is the observed radial velocity of the maser at a measured projected angular distance $\theta$ from the source (this distance is the component measured in the coordinate defined by the semimajor axis of the ring). We also need to know the outer radius of the ring. However, all we can obtain is a lower limit for the outer radius since we cannot tell if the last maser we see is coming from the edge of the ring or if there is material external to this apparent last maser. Another uncertainty comes from the inclination of the ring with respect to the line of sight. We will use the inclination given by Garay et al. (2007), i.e., the ring is almost perpendicular to the plane of the sky. With this in mind, we define the radius of the disk as the angular distance from the central source given by the fit to the slope for the highest radial 


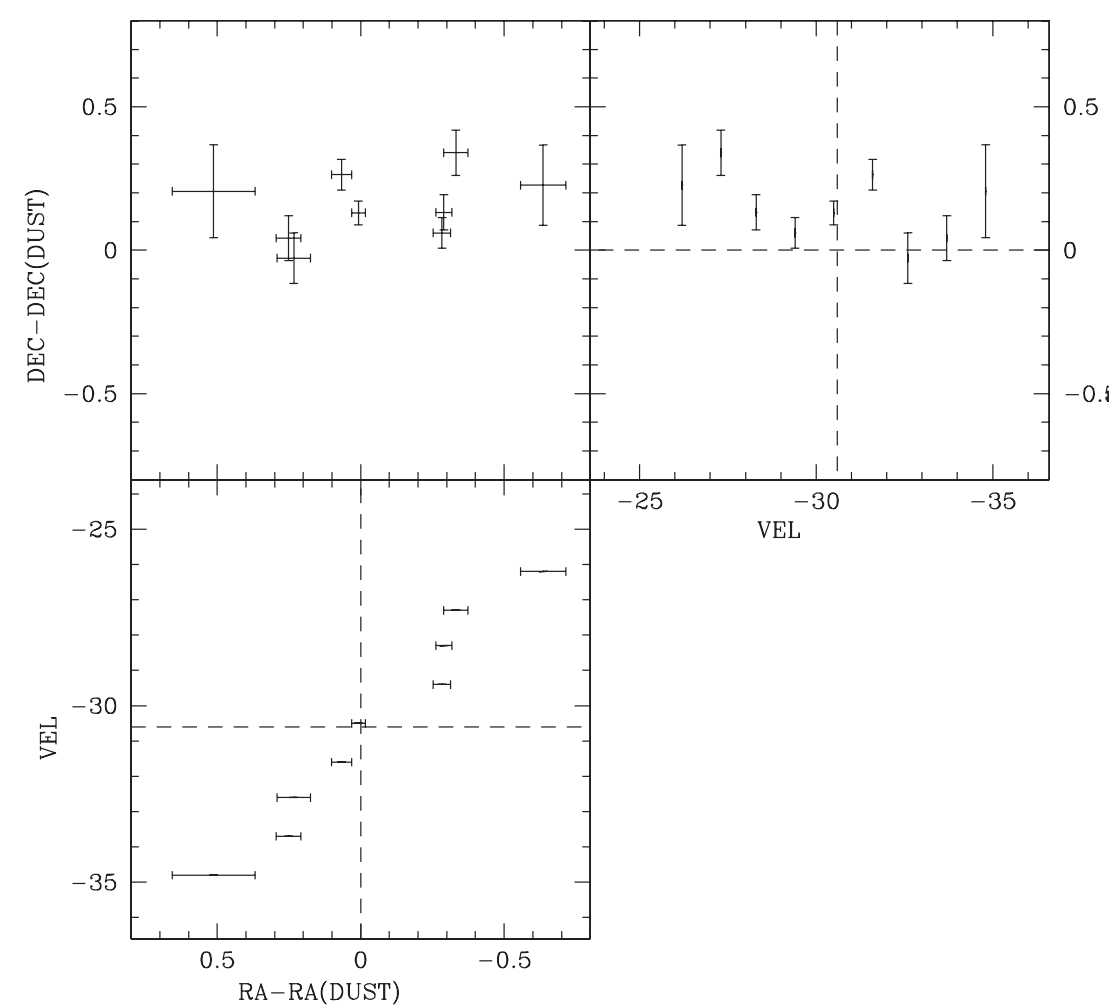

Figure 7. Upper left panel shows the positions of the $\mathrm{SO}_{2}(226.300 \mathrm{GHz})$ emission peaks, measured from each velocity channel and listed in Table 3. The positions are offsets with respect to the peak of the dust continuum. The upper right and lower panels are the position velocity diagrams for the decl. and R.A. directions, respectively.

Table 3

Positions of the Emission Peak in Each Velocity Channel of the $\mathrm{SO}_{2}$ Data

\begin{tabular}{lcccc}
\hline \hline $\begin{array}{c}V_{\mathrm{LSR}} \\
\left(\mathrm{km} \mathrm{s}^{-1}\right)\end{array}$ & $\begin{array}{c}\alpha(\mathrm{J} 2000) \\
16^{\mathrm{h}} 58^{\mathrm{m}}\end{array}$ & $\begin{array}{c}\Delta \alpha \\
(\mathrm{s})\end{array}$ & $\begin{array}{c}\delta(\mathrm{J} 2000) \\
-42^{\circ} 52^{\prime}\end{array}$ & $\begin{array}{c}\Delta \delta \\
(\operatorname{arcsec})\end{array}$ \\
\hline-34.8 & 17.294 & 0.013 & 07.88 & 0.16 \\
-33.7 & 17.270 & 0.004 & 08.04 & 0.08 \\
-32.6 & 17.268 & 0.005 & 08.11 & 0.09 \\
-31.6 & 17.253 & 0.003 & 07.82 & 0.05 \\
-30.5 & 17.248 & 0.002 & 07.95 & 0.04 \\
-29.4 & 17.222 & 0.003 & 08.02 & 0.05 \\
-28.3 & 17.221 & 0.003 & 07.95 & 0.06 \\
-27.3 & 17.217 & 0.004 & 07.74 & 0.08 \\
-26.2 & 17.189 & 0.007 & 07.86 & 0.14 \\
\hline
\end{tabular}

velocity detected. For a velocity of $-54.2 \mathrm{~km} \mathrm{~s}^{-1}$, we obtain $\theta=0.024 \pm 0.002 \mathrm{arcsec}$. The expression for the mass is

$$
M_{\star}=\frac{\theta^{3} D}{G \sin ^{2} i}\left(\frac{\mathrm{d} V}{\mathrm{~d} \theta}\right)^{2},
$$

where $\theta$ is the ring radius, $D$ is the distance to the source, and $i$ is the inclination angle. Introducing the measured values we get a mass

$$
M_{\star}=30 \pm 9 M_{\odot}\left(\frac{D}{2.9 \mathrm{kpc}}\right) .
$$

The error comes mainly from the uncertainties in the radius of the ring and the velocity gradient.

We now briefly discuss the $\mathbf{g} \mathbf{2}$ compact structure of water masers. In Figure 4, we show the relative positions of the water masers in this structure. They distribute in an elongated shape, approximately following the jet direction in that region. This suggests that these masers are tracing the outflow. However, the masers are blueshifted with respect to the systemic velocity of the region, while the $\mathrm{CO}$ outflow is redshifted. There is also a strong velocity gradient in the northernmost part of the structure, suggesting interaction with ambient material.

$$
\text { 4.2. } \mathrm{SO}_{2}
$$

Sulfur-bearing molecules have been studied through millimeter and submillimeter observations of star formation regions. They could account for a significant fraction of the total flux coming from molecules in these regions (Schilke et al. 1997). The chemistry of these molecules has been modeled and its relative abundances have been explored as a possible clock to measure the evolutionary state in low and high-mass star formation regions (Buckle \& Fuller 2003; Hatchell et al. 1998; van der Tak et al. 2003). In the chemical models, the $\mathrm{SO}_{2}$ is the product of reactions from molecules that have been evaporated off the dust grains surfaces (Charnley 1997). There is evidence that the emission originates in outflows (e.g., Codella et al. 2005; Schilke et al. 1997) but also in what look like rotating structures around some sources (Cep A HW2, Jiménez-Serra et al. 2007; AFGL 2591, van der Tak et al. 2006). $\mathrm{SO}_{2}$ also has been observed to trace infall motions (e.g., W51; Sollins et al. 2004).

From the velocity gradient observed in the $\mathrm{SO}_{2}$ transitions we obtain an independent estimate of the mass. In Figure 7, we can see the position velocity diagram showing the position of the emission peak in each velocity channel. This can be interpreted also as gas in a Keplerian motion in a ring around the central source. The estimate of the size of such a ring will have the same uncertainties as described above for the water masers and we will have again a lower limit for the mass estimate. For these data we get a velocity gradient of $-8.8 \pm 0.8 \mathrm{~km} \mathrm{~s}^{-1} \mathrm{arcsec}^{-1}$, and an intercept of $-31.0 \pm 0.3 \mathrm{~km} \mathrm{~s}^{-1}$. The velocity of the most blueshifted detectable $\mathrm{SO}_{2}$ emission is $-34.8 \mathrm{~km} \mathrm{~s}^{-1}$. For this 
velocity, we obtain $\theta=0.43 \pm 0.05$ arcsec. Using Equation (1), we obtain an estimate of the mass given by

$$
M_{\star}=22 \pm 8 M_{\odot}\left(\frac{D}{2.9 \mathrm{kpc}}\right),
$$

where the errors are estimated in the same way that as for the water masers.

Then, the Keplerian masses estimated from the water masers and from the $\mathrm{SO}_{2}$, at physical scales that differ by a factor of $\sim 20$, give consistent large masses for the central star(s). However, the $\mathrm{SO}_{2}$ data set has very limited angular resolution and we find that the apparent gradient seen in Figure 7 can also be modeled as a Gaussian two-component model. From this fit, we find two components separated by $00^{\prime \prime} 74 \pm 0.06$ at a position angle of $109^{\circ} \pm 6^{\circ}$, and with a velocity difference of $2.3 \pm 0.2 \mathrm{~km} \mathrm{~s}^{-1}$. These two components, if assumed to be of negligible mass and located symmetrically with respect to a central object with all the mass (that is not directly detected in our observations), imply a Keplerian mass of $1.6 \pm 0.4 M_{\odot}$, much smaller that the mass derived from the continuous ring model. Alternatively, we can assume that the total mass of the system is distributed between the two components and in this case the Keplerian mass is $12.8 \pm 3.2 M_{\odot}$. Given the limitations of the data, it is very difficult to decide if the $\mathrm{SO}_{2}$ emission is coming from a continuous, ring-like structure or from two discrete molecular clumps.

\subsection{An Accretion Origin for the High Luminosity?}

We have assumed that the high bolometric luminosity, $L_{\text {bol }}=$ $6.2 \times 10^{4} L_{\odot}$, associated with IRAS $16547-4247$ comes mostly from an embedded, massive main-sequence star. This assumption is justified since high-mass objects are expected to quickly evolve to the main sequence, even while accreting and while they are deeply embedded within the dusty core (Zinnecker \& Yorke 2007).

However, an alternative explanation is a lower mass object accreting at a very high rate. In this case, the system would derive most of its luminosity from accretion. The accretion luminosity, $L_{\text {acc }}$, is

$$
L_{\mathrm{acc}}=\frac{G M}{R} \epsilon \dot{M}_{i},
$$

where $G$ is the gravitational constant, $M$ is the mass of the star, $R$ is the radius of the star, $\dot{M}_{i}$ is the infall rate determined from observations al large scales, and $\epsilon$ is the fraction of this large-scale infalling gas that ends being accreted by the star. Assuming that the bolometric luminosity comes mostly from accretion, $L_{\text {acc }} \simeq L_{\text {bol }}$, and that from the observations of Garay et al. (2007) we have that $\dot{M}_{i}=1 \times 10^{-2} M_{\odot} \mathrm{yr}^{-1}$, we derive from Equation (4) a mass-radius relationship that in solar units is

$$
\left[\frac{R}{R_{\odot}}\right]=5.2 \epsilon\left[\frac{M}{M_{\odot}}\right] .
$$

If $\epsilon$ is larger than $\sim 0.2$, we obtain $\left(R / R_{\odot}\right)>\left(M / M_{\odot}\right)$ and since in the main sequence we expect $\left(R / R_{\odot}\right) \simeq\left(M / M_{\odot}\right)$, one would have to conclude that the star is not in the main sequence and most probably is a lower mass object (than the mass value derived from assuming a main-sequence star) accreting at a very high rate and deriving a significant fraction of its total luminosity from accretion. It should be noted, however, that Hosokawa \& Omukai (2009) have argued that massive protostars undergoing strong accretion will have stellar radii an order of magnitude larger than those occurring in the main sequence. Clearly, a better knowledge of $\epsilon$ and of the structure of protostars will help restrict these possibilities and better quantify the contribution of accretion to the total luminosity of forming massive stars.

\section{CONCLUSIONS}

Our main conclusions are as follows.

1. We present VLA $1.3 \mathrm{~cm}$ radio continuum and water maser observations as well as SMA $\mathrm{SO}_{2}(226.300 \mathrm{GHz})$ and $1.3 \mathrm{~mm}$ dust continuum observations toward the massive star formation region IRAS 16547-4247. The $1.3 \mathrm{~cm}$ continuum traces the inner parts of the thermal jet in the region. The $1.3 \mathrm{~mm}$ dust continuum traces a double structure, with component separation of $\sim 2^{\prime \prime}$, with each structure probably marking the position of a star or a stellar group.

2. Water maser emission is present in several distinct parts in the region. The compact structure in group g1 is closely associated with the thermal jet and shows an alignment perpendicular to it. The masers in this group show a velocity gradient that, if interpreted as arising in a Keplerian ring, implies a mass of $\sim 30 M_{\odot}$ for the central star(s).

3. The $\mathrm{SO}_{2}$ emission arises only from the brightest $1.3 \mathrm{~mm}$ dust continuum component. The line emission shows a velocity gradient that, if modeled as a Keplerian ring, gives a mass of $\sim 20 M_{\odot}$, consistent with the mass derived from the $\mathrm{H}_{2} \mathrm{O}$ masers. However, the data can also be fitted with a two-component model that gives smaller Keplerian masses.

We thank an anonymous referee for comments that improved two sections of the paper and for the suggestion of discussing the possibility of significant accretion luminosity. R.F.H. is grateful for support from an SAO predoctoral fellowship. L.F.R. acknowledges the support of CONACyT, México and DGAPA, UNAM. G.G. acknowledges support from CONICYT projects FONDAP no. 15010003 and BASAL PFB-06.

\section{REFERENCES}

Batchelor, R. A., Caswell, J. L., Haynes, R. F., Wellington, K. J., Goss, W. M., \& Knowles, S. H. 1980, Aust. J. Phys., 33, 139

Bonnell, I. A., Bate, M. R., \& Zinnecker, H. 1998, MNRAS, 298, 93 Briggs, D. 1995, PhD thesis, New Mexico Inst. of Mining and Technology Brooks, K. J., Garay, G., Mardones, D., \& Bronfman, L. A. 2003, ApJ, 594, L131

Brooks, K. J., Garay, G., Voronkov, M., \& Rodríguez, L. F. 2007, ApJ, 669, 459

Buckle, J. V., \& Fuller, G. A. 2003, A\&A, 399, 567

Cabrit, S., Goldsmith, P. F., \& Snell, R. L. 1988, ApJ, 334, 196

Charnley, S. B. 1997, ApJ, 481, 396

Cheung, A. C., Rank, D. M., Townes, C. H., Thornton, D. D., \& Welch, W. J. 1969, Nature, 221, 626

Chini, R., Kruegel, E., \& Wargau, W. 1987, A\&A, 181, 378

Codella, C., Bachiller, R., Benedettini, M., Caselli, P., Viti, S., \& Wakelam, V. 2005, MNRAS, 361, 244

Elitzur, M. 1992, Astrophys. Space Sci. Libr., 170, 267

Evans, N. J. 1999, ARA\&A, 37, 311

Forster, J. R., \& Caswell, J. L. 1989, A\&A, 213, 339

Furuya, R. S., Kitamura, Y., Saito, M., Kawabe, R., \& Wootten, H. A. 1999, ApJ, 525,821

Garay, G., Brooks, K. J., Mardones, D., \& Norris, R. P. A. 2003, ApJ, 587, 739

Garay, G., et al. 2007, A\&A, 463, 217

Hatchell, J., Thompson, M. A., Millar, T. J., \& MacDonald, G. H. 1998, A\&A, 338,713

Hosokawa, T., \& Omukai, K. 2009, ApJ, 691, 823

Jiménez-Serra, I., Martín-Pintado, J., Rodríguez-Franco, A., Chandler, C., Comito, C., \& Schilke, P. 2007, ApJ, 661, L187

Jones, B. F., \& Walker, M. F. 1988, AJ, 95, 1755

Kiminki, D. C., et al. 2007, ApJ, 664, 1102 
Lada, C. J. 1991, in The Physics of Star Formation and Early Stellar Evolution, ed. C. J. Lada \& N. D. Kylafis (Dordrecht: Kluwer), 329

McKee, C. F., \& Ostriker, E. C. 2007, ARA\&A, 45, 565

McKee, C. F., \& Tan, J. C. 2002, Nature, 416, 59

Osorio, M., Lizano, S., \& D'Alessio, P. 1999, ApJ, 525, 808

Rodríguez, L. F., Garay, G., Brooks, K. J., \& Mardones, D. 2005, ApJ, 626, 953

Rodríguez, L. F., Moran, J. M., Franco-Hernández, R., Garay, G., Brooks, K. J., \& Mardones, D. 2008, AJ, 135, 2370

Schilke, P., Groesbeck, T. D., Blake, G. A., \& Phillips, T. G. 1997, ApJS, 108, 301

Shu, F. H., Adams, F. C., \& Lizano, S. 1987, ARA\&A, 25, 23

Shu, F.H, Najita, J., Galli, D., Ostriker, E., \& Lizano, S. 1993, in Protostars and Planets III, ed. E. H. Levy \& J. I. Lunine (Tucson, AZ: Univ. Arizona Press), 3
Sollins, P. K., Zhang, Q., \& Ho, P. T. P. 2004, ApJ, 606, 943

Stahler, S. W., Palla, F., \& Ho, P. T. P. 2000, in Protostars and Planets IV, ed. V. Mannings, A. P. Boss, \& S. S. Russell (Tucson, AZ: Univ. Arizona Press), 327

Torrelles, J. M., Patel, N. A., Gómez, J. F., \& Anglada, G. 2002, RevMexAA Conf. Ser., 13, 108

Val'Tts, I. E., Slysh, V. I., Voronkov, M. A., \& Migenes, V. 2005, in ASP Conf. Ser. 340, Future Directions in High Resolution Astronomy, ed. J. Romney \& M. Reid (San Francisco, CA: ASP), 367

van der Tak, F. F. S., Boonman, A. M. S., Braakman, R., \& van Dishoeck, E. F 2003, A\&A, 412, 133

van der Tak, F. F. S., Walmsley, C. M., Herpin, F., \& Ceccarelli, C. 2006, A\&A, 447, 1011

Zinnecker, H., \& Yorke, H. W. 2007, ARA\&A, 45, 481 